Pacific Journal of Mathematics

THE USE OF MITOTIC ORDINALS IN CARDINAL 


\section{THE USE OF MITOTIC ORDINALS IN CARDINAL ARITHMETIC}

\section{ALEXANDER ABIAN}

In this paper, based on the properties of mitotic ordinals, some results of the cardinal arithmetic are obtained in a rather natural way.

In what follows, any reference to order among ordinal numbers is made with respect to their usual order. Thus, if $u$ and $v$ are ordinals then $u \leqq v$ if and only if $u \leqq v$ if and only if " $u \in v$ or $u=v$ ".

Definition. A nonzero ordinal $w$ is called mitotic if and only if it can be partitioned into $\overline{\bar{w}}$ pairwise disjoint subsets each of type w. Such a partition is called a mitotic partition of $w$.

For instance, $\omega$ is a mitotic ordinal since $\omega$ can be partitioned into denumerably many pairwise disjoint denumerable subsets $R_{i}$ with $i=0,1,2, \cdots$, where the elements of $R_{i}$ are precisely the ordinals appearing in the $i$-th row of the following table:

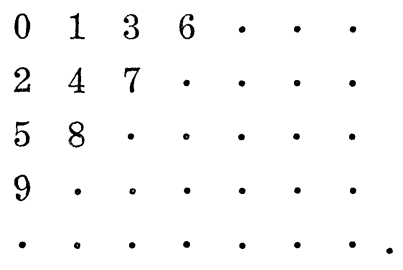

Clearly, each $R_{i}$ is of type $\omega$.

Lemma 1. Let $w$ be a mitotic ordinal. Then $w$ is a limit ordinal. Moreover, for every element $S_{i}$ of a mitotic partition $\left(S_{i}\right)_{i \in w}$ of $w$ we have:

$$
\cup S_{i}=\sup S_{i}=w
$$

Proof. Since $S_{i}$ is of type $w$ we see that $S_{i}$ is similar to $w$. Let $f_{i}$ be a similarity mapping from $w$ onto $S_{i}$. But then by $[1, \mathrm{p} .302]$ we have $x \leqq f_{i}(x)$ for every $x \in w$. Now, assume on the contrary that $w$ is not a limit ordinal and let $k$ be the last element of $w$. But then clearly, $k=f_{i}(k)$ and therefore $k \in S_{i}$. However, since 1 is not a mitotic ordinal, we see that the mitotic partition of $w$ must have at least two distinct elements, $S_{0}$ and $S_{1}$. But then $k \in S_{0}$ and $k \in S_{1}$ which contradicts the fact that $S_{0}$ is disjoint from $S_{1}$. Thus, our assumption is false and $w$ is a limit ordinal. 
Next, since the similarity of $w$ to $S_{i}$ implies the existence of a one-to-one mapping $f_{i}$ from $w$ onto $S_{i}$ such that $x \leqq f_{i}(x)$ for every $x \in w$, we see that $\cup w \leqq S_{i}$ and therefore $\cup w=\cup S_{i}$ since $S_{i} \subseteq$ $w$. On the other hand, since $w$ is a limit ordinal by [1, p. 323] we have $\cup w=w$. Hence, (1) is established.

Based on the natural expansion [1, p. 355] of ordinals we prove the following lemma.

Lemma 2. Let $w$ be a mitotic ordinal and let $\omega^{e} n$ be the last term of the normal expansion of $w$. Then

$$
\overline{\bar{w}}=\overline{\overline{\omega^{e} n}}
$$

Proof. Let $w=u+\omega^{e} n$ and let $\left(S_{i}\right)_{i \in w}$ represent a mitotic partition of $w$. From (1) it follows that for every $i \in w$, we must have $(u+v) \in S_{i}$ for some $v<\omega^{e} n$. But then (2) follows from the fact that $\left(S_{i}\right)_{i \in w}$ is a family of pairwise disjoint elements $S_{i}$.

LemMa 3. For every nonzero ordinal e the ordinal $\omega^{e}$ is mitotic.

Proof. Since $\omega<\omega^{e}$ we see that there is a mitotic ordinal of type $\omega^{h}$ such that $h \leqq e$. Let $P$ be the set of all mitotic partitions of mitotic ordinals of type $\omega^{h}$ which are less than or equal to $\omega^{e}$. Partial order $P$ by $\varliminf^{*}$ as follows:

$$
\left(S_{u_{i}}\right)_{i \in \omega^{u}} \leqq *\left(S_{v_{i}}\right)_{i \in \omega^{v}}
$$

if and only if $S_{u_{i}} \subseteq S_{v_{i}}$ for every $i \in\left(\omega^{u} \cap \omega^{v}\right)$.

Let $\left(\left(S_{u_{i}}\right)_{i \in \omega}\right)_{u \in A}$ be a simply ordered subset of $\left(P, \leqq^{*}\right)$. But then it is easy to verify that $\left(\bigcup_{u \in \cup A} S_{u_{i}}\right)_{i \in \omega} \cup A$ is a mitotic partition of the ordinal $\omega^{\cup A}$. Hence every simply ordered subset of the nonempty partially ordered set $\left(P, \leqq^{*}\right)$ has a least upper bound. Consequently, $\left(P, \leqq^{*}\right)$ has a maximal element $\left(M_{i}\right)_{i \in \omega^{k}}$ where $\omega^{k}$ is a mitotic ordinal such that $k \leqq e$.

Let $\left(M_{i}\right)$ denote the mitotic partition $\left(M_{i}\right)_{i \in \omega^{k}}$ of $\omega^{k}$, i.e.,

$$
\left(M_{i}\right)=\left(M_{i}\right)_{i \in \omega^{k}} \cdot
$$

To prove the lemma it is sufficient to show that $k=e$. Assume on the contrary that $k<e$. Thus $\omega^{k} \omega \leqq \omega^{e}$.

For every $n \in \omega$, let $\left(M_{i}\right) n$ denote the mitotic partition given by (3) where each entry is augmented on the left by $\omega^{k} n$. But then

$\begin{array}{cccc}\left(M_{i}\right) 0 & \left(M_{i}\right) 1 & \left(M_{i}\right) 3 & \text { ・ } \\ \left(M_{i}\right) 2 & \left(M_{i}\right) 4 & \cdot & \text {. } \\ \left(M_{i}\right) 5 & \bullet & \cdot & \text { ・ } \\ \bullet & . & . & \text { • }\end{array}$


is clearly a mitotic partition of $\omega^{k} \omega=\omega^{k+1}$. But since $\omega^{k} \leqq \omega^{k} \omega<$ $\omega^{k+1} \leqq \omega^{e}$ we arrive at a contradiction. Thus, our assumption is false and $k=e$.

LEMma 4. The sum of finitely many pairwise equipollent mitotic ordinals is a mitotic ordinal.

Proof. Obviously, it is sufficient to prove that the sum of two equipollent mitotic ordinals is a mitotic ordinal. Let $\left(R_{i}\right)_{i \in \overline{\bar{u}}}$ and $\left(S_{i}\right)_{i \in \overline{\bar{v}}}$ represent respectively mitotic partitions of mitotic ordinals $u$ and $v$ where $\overline{\bar{u}}=\overline{\bar{v}}=c$. Now, let

$$
R_{i}=\left(r_{0}, r_{1}, r_{2}, \cdots\right) \text { and } S_{i}=\left(s_{0}, s_{1}, s_{2}, \cdots\right) \text {. }
$$

Consider

$$
H_{i}=\left(r_{0}, r_{1}, r_{2}, \cdots,\left(\cup R_{i}\right)+s_{0},\left(\cup R_{i}\right)+s_{1},\left(\cup R_{i}\right)+s_{2}, \cdots\right) .
$$

Clearly, $H_{i} \leqq(u+v)$ and $H_{i}$ is of type $u+v$ for every $i \in c$. But then observing that $\overline{\overline{u+v}}=c$ we see that $\left(H_{i}\right)_{i \in c}$ is a mitotic partition of the ordinal $u+v$. Thus, $u+v$ is mitotic, as desired.

THEOREM 1. An infinite ordinal is mitotic if and only if it is equipollent to the last term of its normal expansion.

Proof. Let $w$ be an infinite ordinal. Without loss of generality we may assume that the normal expansion of $w$ has two terms and is given by:

$$
w=\omega^{a} m+\omega^{e} n .
$$

Now, if $w$ is mitotic then by (2) we see that $w$ is equipollent to the last term of its normal expansion. Conversely, let $w$ be equipollent to the last term of its normal expansion. But then clearly,

$$
\overline{\bar{w}}=\overline{\overline{\omega^{a} m}}=\overline{\overline{\omega^{e} n}} .
$$

However, since $\omega^{a} m$ is a finite sum of summands each equal to $\omega^{a}$, in view of Lemmas 3 and 4 , we see that $\omega^{a} m$ is mitotic. Similarly, $\omega^{e} n$ is mitotic. But then again, from (5), (4) and Lemma 4, we see that $w$ is mitotic, as desired.

From Theorem 1 it follows that each of the following ordinal numbers is mitotic:

$$
\omega^{\omega}, \omega^{\omega}+\omega, \omega_{1}^{\omega}+\omega_{1}, \omega_{2}^{\omega}+\omega_{2} \omega_{1} \omega, \cdots .
$$

Also, since the normal expansion of every infinite cardinal has one term, from Theorem 1, we have: 
COROLlary 1. Every infinite cardinal is mitotic.

Next, based on the properties of mitotic ordinals we derive some results pertaining to the cardinal arithmetic.

THEOREM 2. Let $w$ be a mitotic ordinal and $\left(c_{i}\right)_{i \in w}$ a nondecreasing sequence of type $w$ of cardinals $c_{i}$. Then

$$
\prod_{i \in w} c_{i}=\left(\prod_{i \in w} c_{i}\right)^{\overline{\bar{w}}} .
$$

Proof. Let $\left(S_{i}\right)_{i \in w}$ be a mitotic partition of $w$. Since $\left(c_{i}\right)_{i \in w}$ is nondecreasing, we have

$$
\prod_{i \in w} c_{i} \leqq \prod\left\{c_{i} \mid c_{i} \in S_{j}\right\} \text { for every } j \in w
$$

and since the right side of the above inequality is a subproduct of the left side, we have

$$
\prod_{i \in w} c_{i}=\prod\left\{c_{i} \mid c_{i} \in S_{j}\right\} \text { for every } j \in w .
$$

On the other hand, in view of the general commutativity and associativity of the infinite product of cardinal numbers, we have

$$
\prod_{i \in w} c_{i}=\prod_{j \in w}\left(I I c_{i} \mid c_{i} \in S_{j}\right\} .
$$

But then (6) follows readily from (7) and (8).

Based on Theorem 2, we prove a theorem which extends a result of Tarski-Hausdorff [2, p. 14] to the case of a nondecreasing sequence of cardinals.

THEOREM 3. Let $w$ be a mitotic ordinal and $\left(c_{i}\right)_{i \in w}$ a nondecreasing sequence of type $w$ of nonzero cardinals $c_{i}$. Then

$$
\prod_{i \in w} c_{i}=\left(\sup _{i \in w} c_{i}\right)^{\overline{\bar{w}}}
$$

Proof. Since $c_{i} \leqq \sup _{i \in w} c_{i}$ for every $i \in w$, we have

$$
\prod_{i \in w} c_{i} \leqq\left(\sup _{i \in w} c_{i}\right)^{\overline{\bar{w}}} .
$$

On the other hand, for establishing (9), we may assume without loss of generality, that $c_{i}>1$ for every $i \in w$. But then we have:

$$
\left(\sup _{i \in w} c_{i}\right)^{\overline{\bar{w}}} \leqq\left(\sum_{i \in w} c_{i}\right)^{\overline{\bar{w}}} \leqq\left(\sum_{i \in w} c_{i}\right)^{\overline{\bar{w}}}
$$

and then (9) follows readily from (6), (10) and (11). 
Thus, Theorem 3 is proved.

Let us observe that the formula analogous to (9) for the sum of an (not necessarily nondecreasing) infinite sequence $\left(c_{i}\right)_{i \in v}$ of type $v$ (not necessarily mitotic) of nonzero cardinals $c_{i}$ is given by:

$$
\sum_{i \in v} c_{i}=\overline{\bar{v}} \sup _{i \in v} c_{i}
$$

REMARK. In the arithmetic of ordinal numbers infinite sums and products of ordinals are respectively equal to the limit of their partial sums and partial products. In fact, in ordinal arithmetic, evaluation of the result of an infinite operation as the limit of those of partial ones is a general method. In contrast to this, in the arithmetic of cardinal numbers infinite sums and products of cardinals are not equal, in general, to the limit of their partial sums and the limit of their partial products respectively. However, as shown below, in cardinal arithmetic, infinite sums of cardinals and products of nondecreasing cardinals are respectively equal to the sum of their partial sums and to the product of their partial products (this, in general, is not true in ordinal arithmetic).

The statement concerning an infinite sum of cardinals can be given as a corollary of (12).

Corollary 2. Let $\left(c_{i}\right)_{i \in v}$ be an infinite sequence of type $v$ of nonzero cardinals $c_{i}$. Then

$$
\sum_{i<v} c_{i}=\sum_{u<v}\left(\sum_{i \leqq u} c_{i}\right)
$$

Proof. From (12) it follows:

$$
\sum_{u<v}\left(\sum_{i \leq u} c_{i}\right)=\sum_{u<v} \overline{\bar{u}} \cdot c_{u}=\overline{\bar{v}} \cdot \overline{\bar{v}} \sup c_{i}=\overline{\bar{v}} \sup c_{i}=\sum_{i<v} c_{i} .
$$

Next, based on the properties of mitotic ordinals we prove the following theorem.

THEOREM 4. Let $u$ be limit ordinal and $\left(c_{i}\right)_{i \in u}$ a nondecreasing sequence of type $u$ of cardinals $c_{i}$. Then

$$
\prod_{i<u} c_{i}=\prod_{j<u}\left(\prod_{i<j} c_{i}\right)
$$

Proof. Without loss of generality, we may assume that the normal expansion of $u$ has two terms and is given by

$$
u=\omega^{e} p+\omega^{h} q .
$$

Hence, by Lemma 3, without loss of generality, we may assume 
that $u$ is a sum of two mitotic ordinals $w$ and $r$, i.e.

$$
u=w+r \text { with } \overline{\bar{w}} \geqq \overline{\bar{r}} \geqq \boldsymbol{S}_{0} .
$$

Thus, to prove (14), it is enough to show that

$$
\prod_{i<w+r} c_{i}=\prod_{i<w+r}\left(\prod_{i<j} c_{i}\right) .
$$

However, since $u$ is a limit ordinal and $c_{j} \leqq \Pi_{i<j+1} c_{i}$ for every $j<u$, we see that the left side of the equality sign in (16) is less than or equal to the right side. Thus, it is enough to show that the right side is less than or equal to the left side.

Since $w$ and $r$ are both mitotic ordinals, in view of (15) and (9) we have:

$$
\begin{aligned}
\prod_{j<w+r}\left(\prod_{i<j} c_{i}\right) & =\prod_{i<w}\left(\prod_{i<j} c_{i}\right) \cdot \prod_{j<r}\left(\prod_{i<w+j} c_{i}\right) \\
& \leqq\left(\sup _{i<w} c_{i}\right)^{\overline{\bar{w}} \cdot \overline{\bar{w}}} \cdot \prod_{j<r}\left(\prod_{j<w} c_{i} \cdot \prod_{i<j} c_{w+i}\right) \\
& \leqq\left(\sup _{i<w} c_{i}\right)^{\overline{\bar{w}}} \cdot\left(\sup _{i<w} c_{i}\right)^{\overline{\bar{w}} \cdot \overline{\bar{r}}} \cdot\left(\sup _{i<r} c_{w+i}\right)^{\bar{r}} \overline{\bar{r}} \\
& =\left(\sup _{i<w} c_{i}\right)^{\overline{\bar{w}}} \cdot\left(\sup _{i<r} c_{w+i}\right)^{\overline{\bar{r}}} \\
& =\prod_{i<w} c_{i} \cdot \prod_{i<r} c_{w+i}=\prod_{i<w+r} c_{i}
\end{aligned}
$$

as desired.

Finally, based on (14) we obtain the formula analogous to (13) for the product of cardinals.

THEOREM 5. Let $\left(c_{i}\right)_{i<v}$ be an infinite nondecreasing sequence of type $v$ of cardinals $c_{i}$. Then

$$
\prod_{i<v} c_{i}=\prod_{\jmath<v}\left(\prod_{i \leq \jmath} c_{i}\right) .
$$

Proof. As the proof indicates, without loss of generality we may assume $v=u+1$ where $u$ is a limit ordinal. But then from (14) it follows:

$$
\prod_{i<u+1} c_{i}=\left(\prod_{i<u} c_{i}\right) c_{u}=\prod_{j<u}\left(\prod_{i<j} c_{i}\right) \cdot c_{u}=\prod_{j<u+1}\left(\prod_{i \leq j} c_{i}\right) .
$$

\section{REFERENCES}

1. A. Abian, Theory of Sets and Transfinite Arithmetic, W. B. Saunders (1965).

2. A. Tarski, Quelques théorèmes sur les alephs, Fund. Math., 7 (1925), 1-14.

Received April 19, 1971. 


\section{PACIFIC JOURNAL OF MATHEMATICS}

\section{EDITORS}

\section{H. SAMElson}

Stanford University

Stanford, California 94305

C. R. Новву

University of Washington

Seattle, Washington 98105
J. DugunduI

Department of Mathematics University of Southern California

Los Angeles, California 90007

RICHARD ARENS

University of California

Los Angeles, California 90024

\section{ASSOCIATE EDITORS}
E. F. BECKENBACH
B. H. NeUmanN
F. WOLF
K. YosHIDA

\section{SUPPORTING INSTITUTIONS}

UNIVERSITY OF BRITISH COLUMBIA

UNIVERSITY OF SOUTHERN CALIFORNIA

CALIFORNIA INSTITUTE OF TECHNOLOGY

STANFORD UNIVERSITY

UNIVERSITY OF CALIFORNIA

UNIVERSITY OF TOKYO

MONTANA STATE UNIVERSITY

UNIVERSITY OF UTAH

UNIVERSITY OF NEVADA

WASHINGTON STATE UNIVERSITY

NEW MEXICO STATE UNIVERSITY

OREGON STATE UNIVERSITY

UNIVERSITY OF OREGON

OSAKA UNIVERSITY

UNIVERSITY OF WASHINGTON

* *

AMERICAN MATHEMATICAL SOCIETY

NAVAL WEAPONS CENTER 


\section{Pacific Journal of Mathematics}

\section{Vol. 43, No. $1 \quad$ March, 1972}

Alexander (Smbat) Abian, The use of mitotic ordinals in cardinal

arithmetic ....................................... 1

Helen Elizabeth. Adams, Filtrations and valuations on rings ......... 7

Benno Artmann, Geometric aspects of primary lattices .............. 15

Marilyn Breen, Determining a polytope by Radon partitions ........... 27

David S. Browder, Derived algebras in $L_{1}$ of a compact group .......... 39

Aiden A. Bruen, Unimbeddable nets of small deficiency .............. 51

Michael Howard Clapp and Raymond Frank Dickman, Unicoherent

compactifications ............................... 55

Heron S. Collins and Robert A. Fontenot, Approximate identities and the strict topology ................................... 63

R. J. Gazik, Convergence in spaces of subsets................. 81

Joan Geramita, Automorphisms on cylindrical semigroups ........... 93

Kenneth R. Goodearl, Distributing tensor product over direct product ..... 107

Julien O. Hennefeld, The non-conjugacy of certain algebras of

operators ................................... 111

C. Ward Henson, The nonstandard hulls of a uniform space ........... 115

M. Jeanette Huebener, Complementation in the lattice of regular

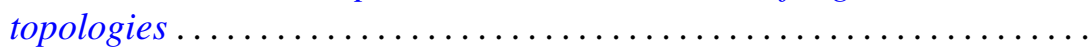

Dennis Lee Johnson, The diophantine problem $Y^{2}-X^{3}=A$ in a

polynomial ring .................................... 151

Albert Joseph Karam, Strong Lie ideals . . . . . . . . . . . . . . . . . . . . 157

Soon-Kyu Kim, On low dimensional minimal sets ............... 171

Thomas Latimer Kriete, III and Marvin Rosenblum, A Phragmén-Lindelöf

theorem with applications to $M(u, v)$ functions ..... . .

William A. Lampe, Notes on related structures of a universal algebra . . . . 189

Theodore Windle Palmer, The reducing ideal is a radical .

207

Kulumani M. Rangaswamy and N. Vanaja, Quasi projectives in abelian and module categories ................................ 221

Ghulam M. Shah, On the univalence of some analytic functions ......... 239

Joseph Earl Valentine and Stanley G. Wayment, Criteria for Banach

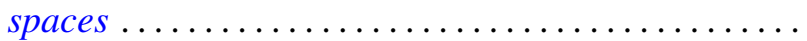

Jerry Eugene Vaughan, Linearly stratifiable spaces ............... 253

Zbigniew Zielezny, On spaces of distributions strongly regular with respect to partial differential operators ..................... 